\title{
Research Article \\ Study of Structural and Phase Transition of Nickel Metal
}

\author{
Pham Huu Kien \\ Thai Nguyen University of Education, No. 20 Luong Ngoc Quyen, Thai Nguyen, Vietnam \\ Correspondence should be addressed to Pham Huu Kien; phkien80@gmail.com
}

Received 23 December 2013; Accepted 14 January 2014; Published 9 March 2014

Academic Editors: M. Fantini and S. Sombra

Copyright (C) 2014 Pham Huu Kien. This is an open access article distributed under the Creative Commons Attribution License, which permits unrestricted use, distribution, and reproduction in any medium, provided the original work is properly cited.

\begin{abstract}
Annealing study of nickel metal in the temperature range $300-1000 \mathrm{~K}$ has been carried out using molecular dynamics (MD) simulations. The simulation is done for models containing $10^{4}$ particles $\mathrm{Ni}$ at both crystalline and amorphous states. We obtain the change as a function of annealing time for the potential energy of system, pair radial distribution function (PRDF), and distribution of coordination number (DCN). The calculation shows that the aging slightly reduces the potential energy of system. This result evidences that the amorphous model undergoes different quasiequilibrated states during annealing. The crystalline model undergoes the slow relaxation which reduces the energy of system and eliminates structural defects in crystal lattices.
\end{abstract}

\section{Introduction}

The structure and atomic mechanism of glass formation from liquid metal have been under intensive study from both experiment and computer simulation. By using different diffraction techniques as X-ray and neutron diffraction or turning electronic microscopic, experiment data has provided the important information of structural general arrangement of metals through the RDF and structure factor. Both functions have splitting second peak, which was usually thought to be related to the existence of local icosahedral in amorphous state [1-7]. Based on the MD simulation, Liu et al. [6] suggested that a laboratory or simulated metallic glass is generally a distorted metallic solid including a significant amount of icosahedral, each consisting of 13 atoms; that is, every atom in metallic glass has 12 atoms in its first-neighbor shell to form an icosahedral. On the other hand, Lopez suggested that the shoulder of RDF indicates the presence of two displaced atoms. At higher temperature this presence is washed out by thermal averaging [7]. However, the atomic mechanism of amorphous formation from liquid state is still not well understood. In addition, we found only few works concerning this problem. In addition, our understanding of the atomic mechanism in crystalline and liquid states is still limited. From MD simulation study, Solhjoo et al. showed that during solidification of aluminum, a crystalline and amorphous-like structure is formed depending on the cooling rate [8]. Ozgen and Duruk [9] showed that when the slow cooling process is applied continuously, not gradually, the systems firstly have a tendency of complex arrangement in cluster level at the glassy transition temperature, and, again, it returned to liquid state since they have an energetically unfavorable structure. Sarkar et al. revealed that the induced pressure during solidification takes the aluminum in crystalline form even in very high cooling rate [10]. However, the atomic mechanism of crystallization from liquid metal has not yet been fully investigated.

Therefore, the main purpose of present paper is to indicate the atomic mechanism of crystallization and amorphous formation in nickel metal using the pair RDF, coordination number distribution, simplex statistics, and 3D visualization. The paper is organized as follows. After a brief introduction we describe in detail the simulation technique in Section 2. The structural characteristics, crystallization, amorphous formation, and estimating of thermodynamic properties are presented in Section 3. Section 4 is devoted to the main conclusions.

\section{Calculation Procedure}

We consider a system of single-component atoms that interact mutually through the Pak-Doyama potential [11-14]:

$$
\begin{aligned}
U\left(r_{i j}\right)= & -0.12929\left(r_{i j}-1.82709\right)^{4}+1.16473\left(r_{i j}-2.50849\right)^{2} \\
& -0.135705 ; \quad r_{i j}<3.44 \AA .
\end{aligned}
$$




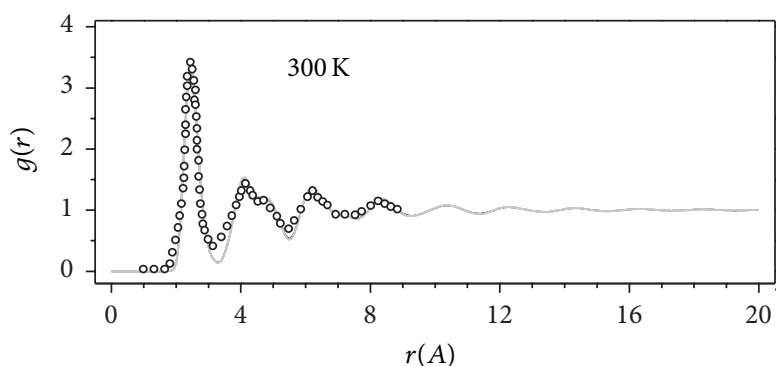

$\begin{array}{ll}-10^{6} \text { steps } & 7 \times 10^{6} \text { steps } \\ -3 \times 10^{6} \text { steps } \quad \circ \quad \text { Experimental data of T. Ichikawa (1975) }\end{array}$

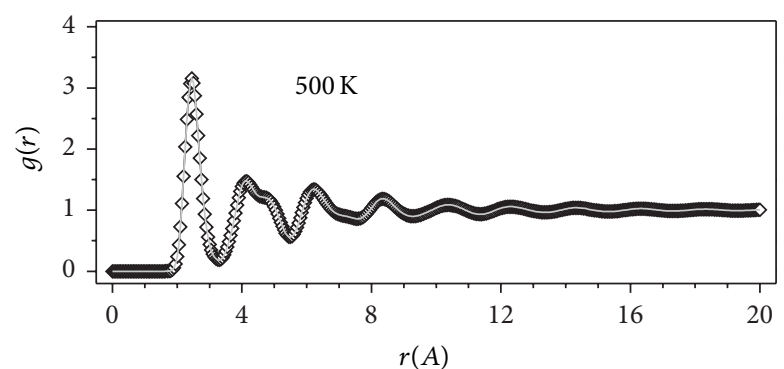

$-10^{6}$ steps

$\diamond 3 \times 10^{6}$ steps $7 \times 10^{6}$ steps

Figure 1: The RDF for 300- and 500- model.
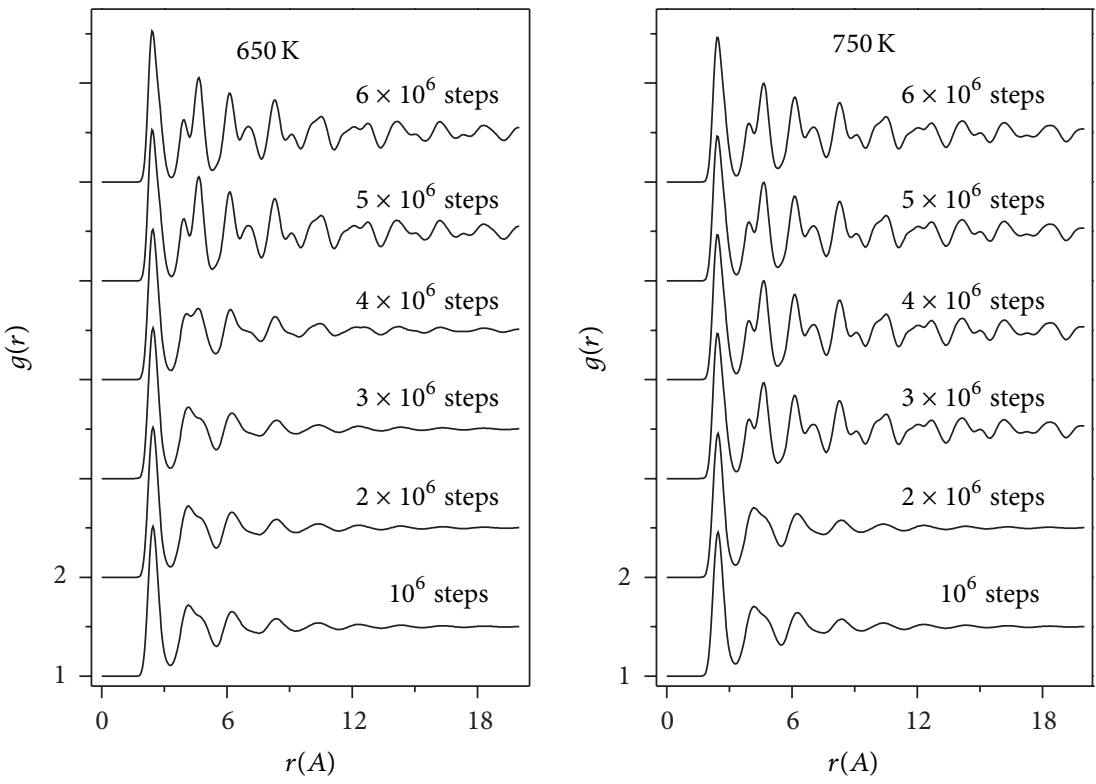

FIgURE 2: The RDF for 650- and 750-model.
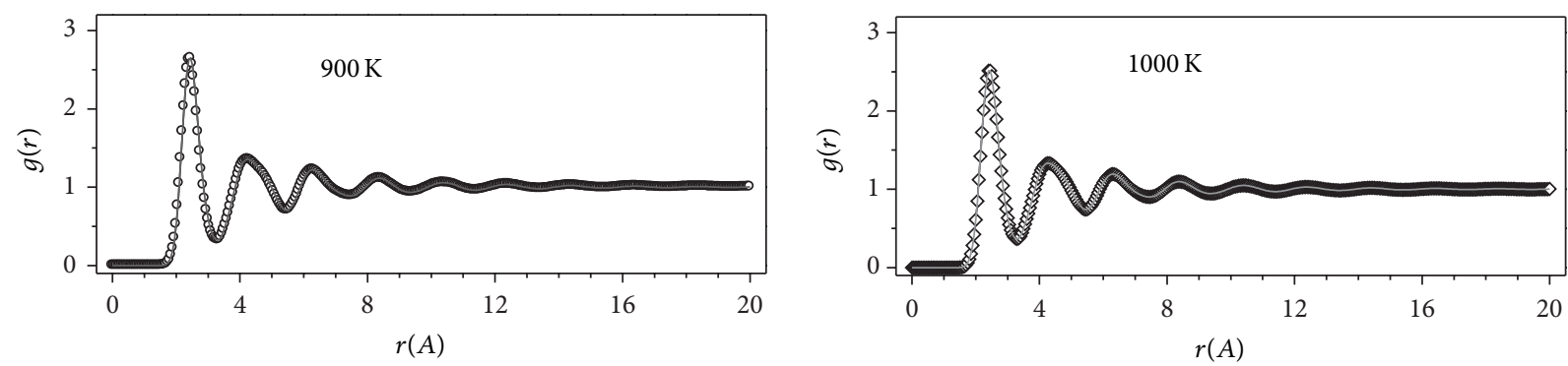

$\begin{array}{ll}- & 10^{6} \text { steps } \\ \circ \quad & 3 \times 10^{6} \text { steps } \\ -\quad 7 \times 10^{6} \text { steps }\end{array}$

$$
\begin{aligned}
& -10^{6} \text { steps } \\
& \prec \quad 3 \times 10^{6} \text { steps } \\
& -\quad 7 \times 10^{6} \text { steps }
\end{aligned}
$$

FIGURE 3: The RDF for 900- and 1000-model. 


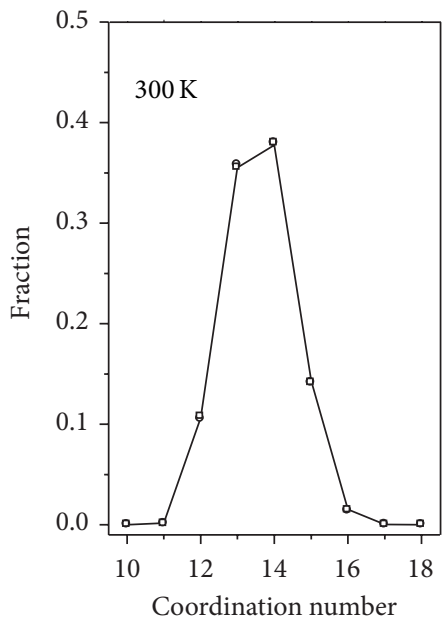

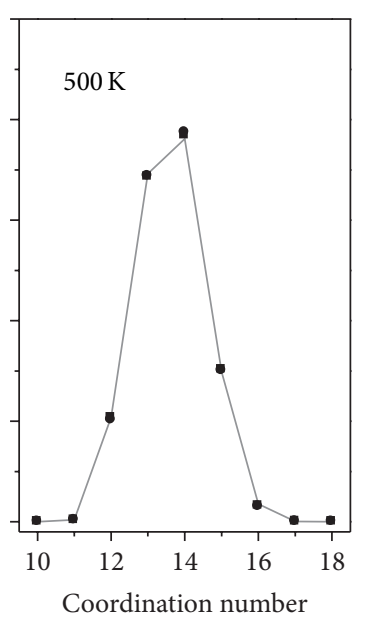

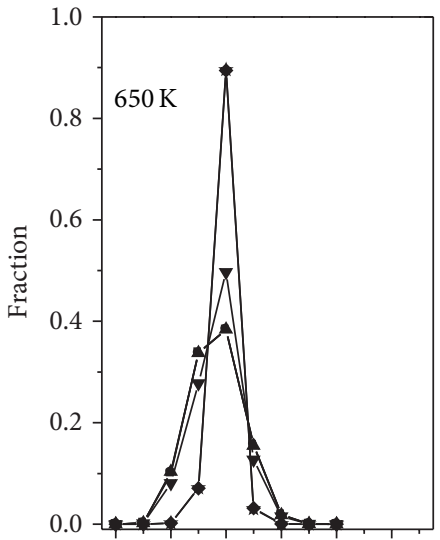

$\begin{array}{llllll}10 & 12 & 14 & 16 & 18 & 20\end{array}$

Coordination number

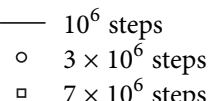

$\rightarrow 10^{6}$ steps

$\rightarrow 2 \times 10^{6}$ steps

$\simeq 3 \times 10^{6}$ steps
- $10^{6}$ steps

- $3 \times 10^{6}$ steps

- $7 \times 10^{6}$ steps

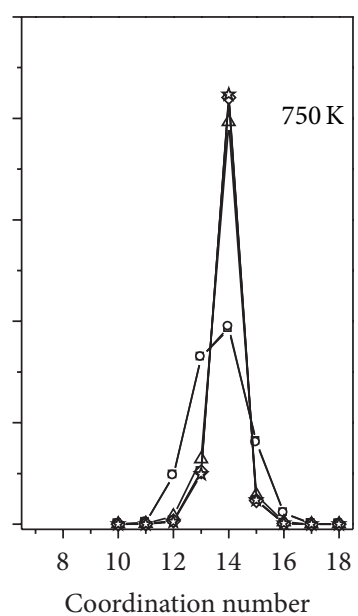

$\rightarrow-4 \times 10^{6}$ steps

$\multimap 5 \times 10^{6}$ steps

$\star 6 \times 10^{6}$ steps
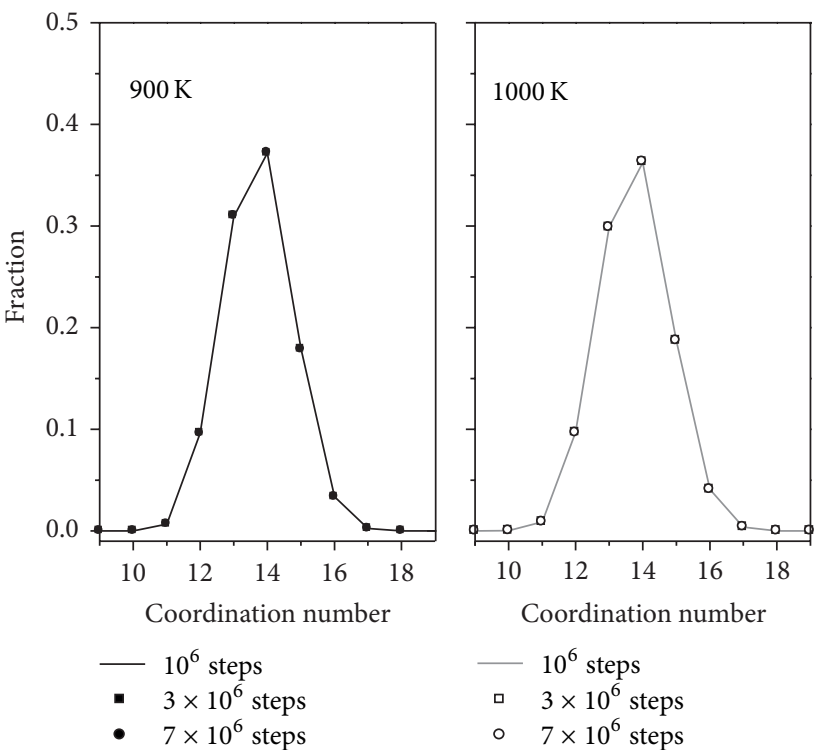

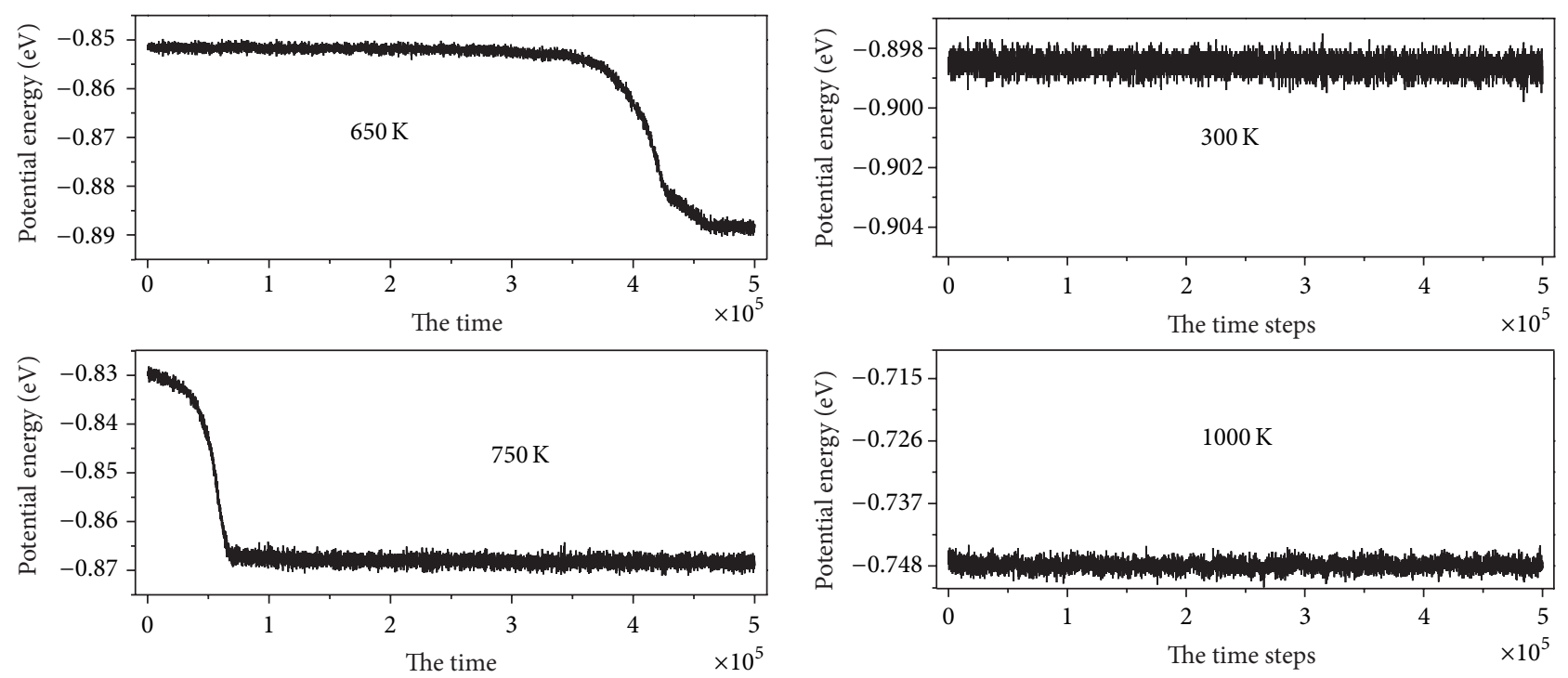

FIgURE 5: The dependence of potential energy as a function of annealing time for obtained models.

nickel. For 300- and 500-model no aging effect on PRDF was found. The PRDFs are almost unchanged over whole time. Hence the structure of considered models remains amorphous for the time scale of simulation. It seems that the aging effect is difficult to detect by the averaged quantities like PRDF. However, unlike the above two models, a significant change in PRDFs is observed for 650- and 750-model. In particular, as shown in Figure 2, the intensities of second peak and several other ones noticeably increase with the annealing time. Moreover, new peaks located at large distances appeal. This result indicates that the observed PRDFs do not resemble the amorphous structure, but it represents another one which is, as shown below, the crystalline structure. As shown in Figure 3, for 900- and 1000-models, PRDFs resemble the liquid structure. The above analysis reveals that for nickel metal there are structural phases (liquid, crystal, and amorphous phase) in the temperature ranging from 300 to $1000 \mathrm{~K}$.

The aging effect can be detected through the distribution of coordination number (DCN). The simulation result on DCN is shown in Figure 4. For low-temperature models (300and 500-model) one can see a pronounced peak located at the point 13 . The height of the peak is about 0.4 and the DCN in general is unchanged with annealing time. Meanwhile, for 650- and 750-model DCN strongly varies. Within a relatively short time the height and location of the peak of DCN are 0.4 and 13 , respectively. After annealing of $2 \times 10^{7}$ steps the height of DCN peak increases up to 0.6 and its location shifts to 14. This result clearly evidences the structural transformation in high-temperature models. For high-temperature models (900- and 1000-model), the DCN in general is unchanged with annealing time.

Further information about the aging effect is inferred from the potential energy of system during annealing process. As shown in Figure 5 the energy for low-temperature models (300- and 500-model) and high-temperature models (900- and 1000-model) oscillated around a defined value. Although the amplitude of these fluctuations is large, it is clear that the energy has a tendency to slightly decrease with annealing time. This means that the system stays in metastable states over whole time and spontaneously transits to more stable states, that is, to the state having smaller potential energy. In principle, the system can reach the equilibrium upon infinite long annealing. However, the time requested is too large so that we do not observe it in the simulation. For 650- and 750-model the energy of system initially oscillated around some value like the case of low-temperature model, but after moderated time it rapidly is dropped to a much lower value. The energy decrease is about $0.042 \mathrm{eV}$ for both 650 and 750-model. The close energy decrease for two models evidences the transformation from amorphous into the similar crystalline structure. With longer annealing the energy of system again oscillates around a new defined value (see Figure 5). This result clearly shows that the system undergoes three stages. At first stage although the relaxation proceeds fast, the models structure remains amorphous and only the energy of system varies. Within the second stage a structural transformation occurs. The energy of system is dropped and the structural characteristics such as PRDF and DCN strongly vary. The amorphous structure now transforms into the crystalline. The last stage is the relaxation of crystalline model. Like first stage the energy has a tendency to slightly reduce which relates to the elimination of structural defects in crystalline lattices.

The crystalline structure can be seen from the snapshot of particles arrangement in the simulation box which is shown in Figure 6. Here one can see the amorphous structure for the short-time annealing model and crystalline for long annealing model. The crystal in the obtained model resembles the bcc lattice which has 8 nearest neighbors and 4 others at 


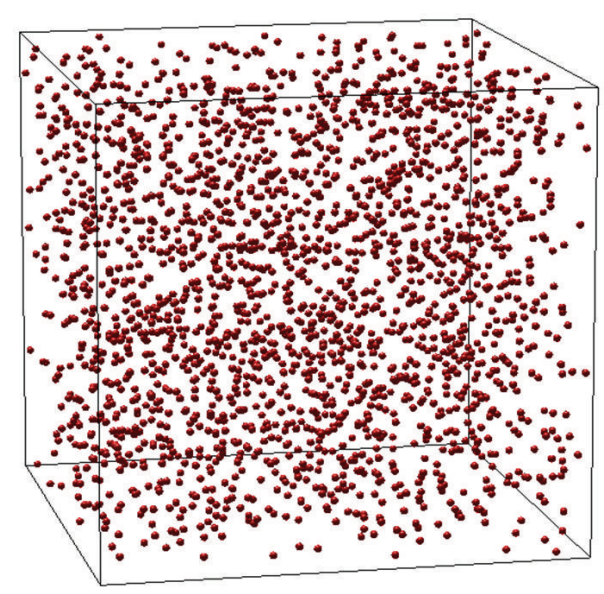

(a)

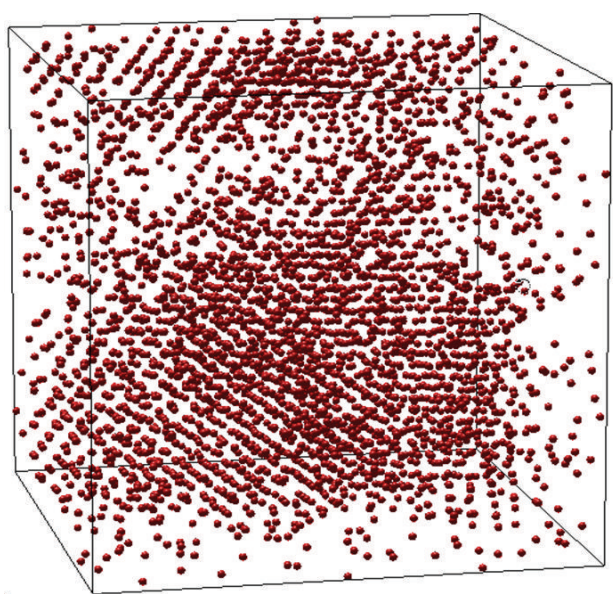

(b)

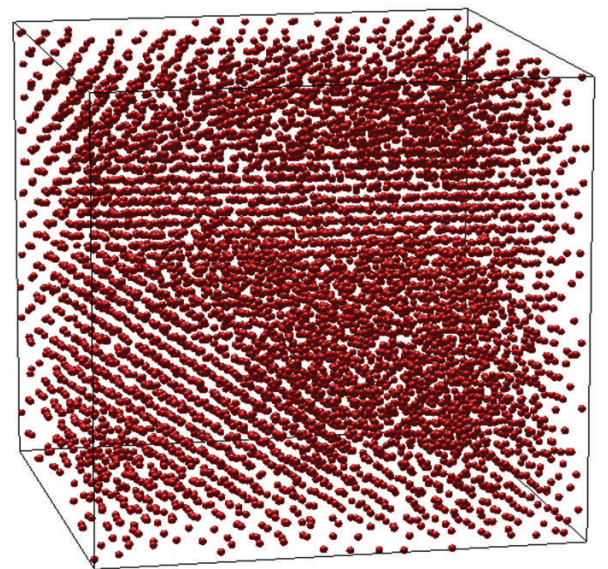

(c)

FigURE 6: 3D visualization of nickel atoms arrangement for $750 \mathrm{~K}$ models: (a), (b), and (c) represent the models with relaxation time of $10^{6}$, $3 \times 10^{6}$, and $7 \times 10^{6} \mathrm{MD}$ steps.

the next coordination sphere. One can see that the atoms arrangement strongly depends on the relaxation time and rate. For the case of nickel metal, the atoms arrangement resembles the crystalline structure after the sample is relaxed by $7 \times 10^{6} \mathrm{MD}$ steps with the relaxation rate of $4 \times 10^{-14} \mathrm{~s}$.

\section{Conclusion}

In this paper we have investigated the aging effect for bulk nickel at temperatures ranging from 300 to $1000 \mathrm{~K}$. The result reveals that the rapidly-quenched bulk models are far from the equilibrium for the relatively long annealing time of $10^{6}-3 \times 10^{7} \mathrm{MD}$ steps. When the models have been annealed at low temperatures, their structure remains amorphous for annealing times much longer than the time scale of simulation. The structural characteristics such as PRDF and DCN are almost unchanged during annealing process. The aging causes only small local rearrangement of particles leading to slightly decreasing the potential energy of system. It means that the system spontaneously undergoes different quasiequilibrated states. As the model has been annealed at high temperatures we observe the crystallization of amorphous solid nickel. The system undergoes the following stages: the relaxation of amorphous model; the transformation from amorphous into the crystalline state which causes a significant change in PRDF, DCN, and the potential energy of system; the relaxation of crystalline model.

\section{Conflict of Interests}

The author declares that there is no conflict of interests regarding the publication of this paper.

\section{Acknowledgment}

The author is grateful to Professor P. K. Hung for many enlightening discussions on the problems discussed here. 
This work was supported in part by Projects DH-2014 of Thai Nguyen University.

\section{References}

[1] Y. Waseda and S. Tamaki, "Structures of 3d-transition metals in the liquid state," Philosophical Magazine, vol. 32, no. 2, pp. 273281, 1975.

[2] P. K. Leung and J. G. Wright, "Structural investigations of amorphous transition element films: II. Chromium, iron, manganese and nickel," Philosophical Magazine, vol. 30, no. 5, pp. 995-1108, 1974.

[3] T. Ichikawa, "The assembly of hard spheres as a structure model of amorphous iron," Physica Status Solidi (A) Applied Research, vol. 29, no. 1, pp. 293-302, 1975.

[4] Y. Waseda, "The structure of liquids, amorphous solids and solid fast ion conductors," Progress in Materials Science, vol. 26, pp. 1$122,1981$.

[5] T. Egami and Y. Waseda, "Atomic size effect on the formability of metallic glasses," Journal of Non-Crystalline Solids, vol. 64, no. 1-2, pp. 113-134, 1984.

[6] R. S. Liu, D. W. Qi, and S. Wang, "Subpeaks of structure factors for rapidly quenched metals," Physical Review B, vol. 45, p. 451, 1991.

[7] J. M. Lopez and M. Silbert, "Structural diffusion model calculations of the pair distribution function of aluminium: from the liquid to the amorphous phase," Solid State Communications, vol. 69 , no. 5 , pp. 585-587, 1989.

[8] S. Solhjoo, A. Simchi, and H. Aashuri, "Molecular dynamics simulation of melting, solidification and remelting processes of aluminum," Transaction of Mechanical Engineering, vol. 36, no. 13, 2012.

[9] S. Ozgen and E. Duruk, "Molecular dynamics simulation of solidification kinetics of aluminium using Sutton-Chen version of EAM," Materials Letters, vol. 58, no. 6, pp. 1071-1075, 2004.

[10] A. Sarkar, P. Barat, and P. Mukherjee, "Molecular dynamics simulation of rapid solidification of aluminum under pressure," International Journal of Modern Physics B, vol. 22, no. 17, pp. 2781-2785, 2008.

[11] V. V. Hoang, "Computer simulation of the effects of B and P concentrations on microstructure in amorphous Fe-B and Fe-P alloys," Physica B, vol. 348, no. 1-4, pp. 347-352, 2004.

[12] P. K. Hung and P. H. Kien, "New model for tracer-diffusion in amorphous solid," European Physical Journal B, vol. 78, no. 1, pp. 119-125, 2010.

[13] P. K. Hung, P. H. Kien, and L. T. Vinh, "Evidence of "microscopic bubbles" and a new diffusion mechanism for amorphous alloys," Journal of Physics Condensed Matter, vol. 22, no. 3, Article ID 035401, 2010.

[14] V. V. Hoang and N. H. Cuong, "Local icosahedral order and thermodynamics of simulated amorphous Fe," Physica B: Condensed Matter, vol. 404, no. 2, pp. 340-346, 2009. 

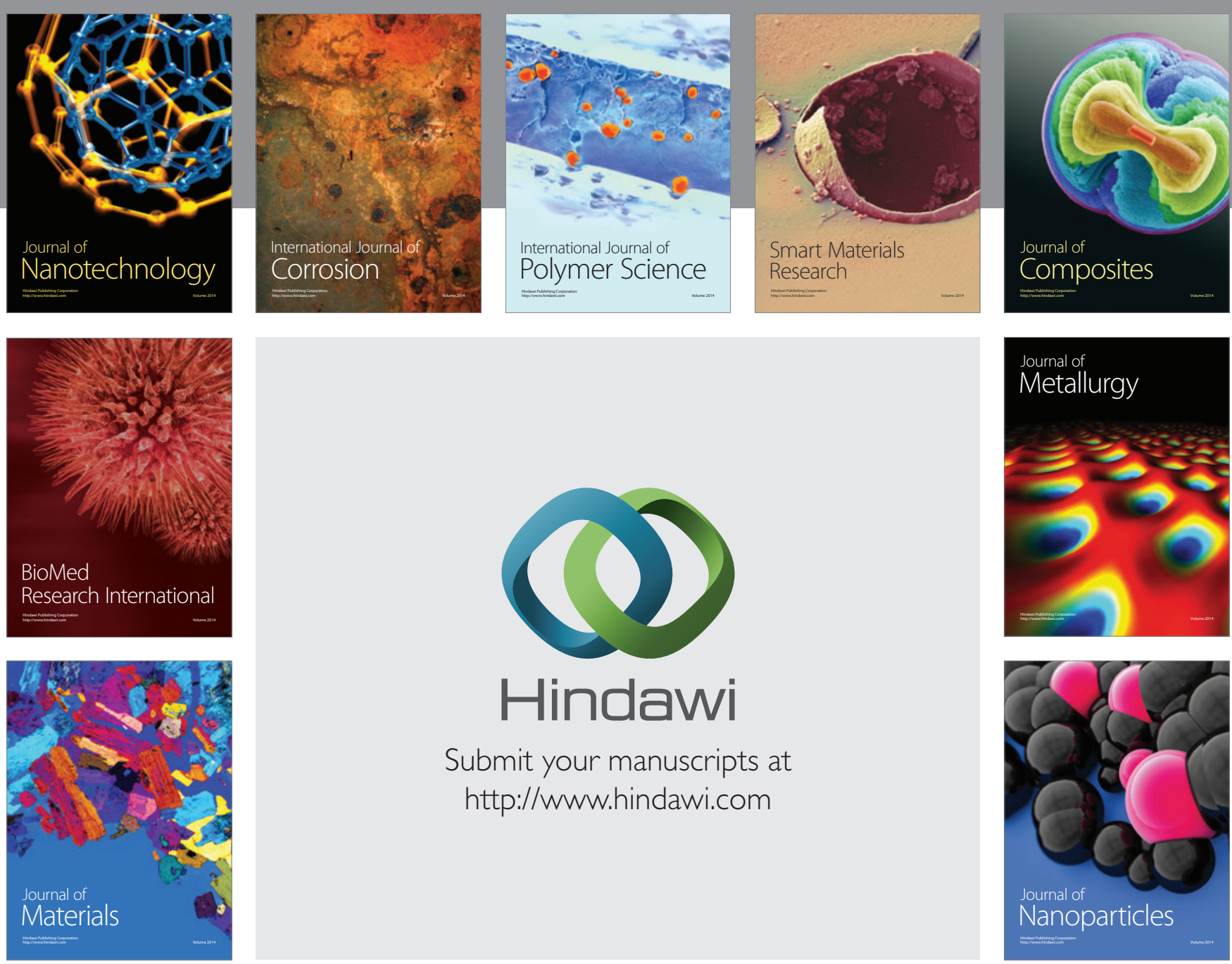

Submit your manuscripts at http://www.hindawi.com
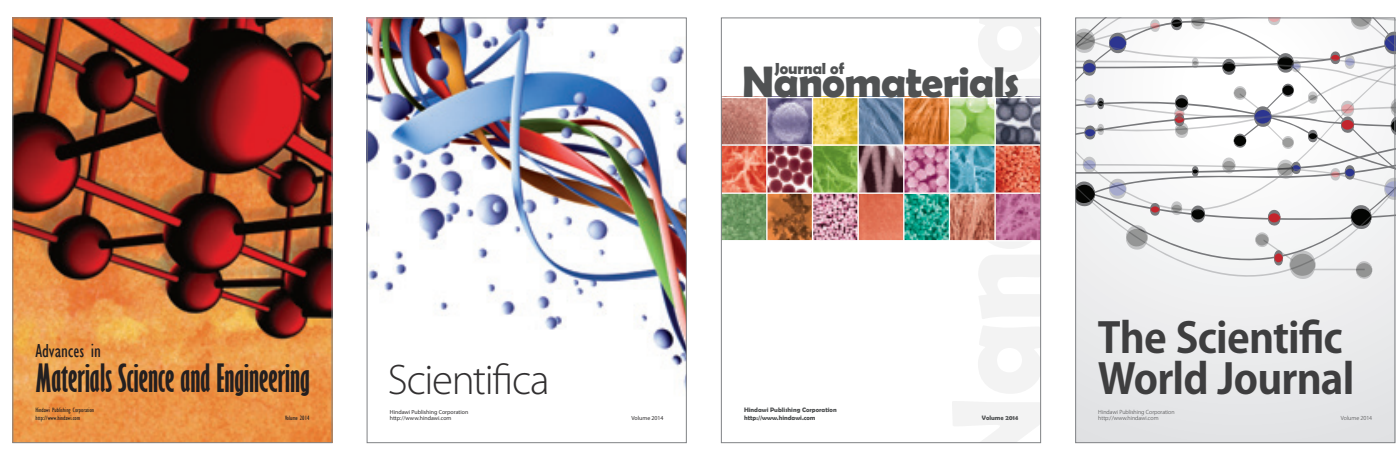

\section{The Scientific World Journal}
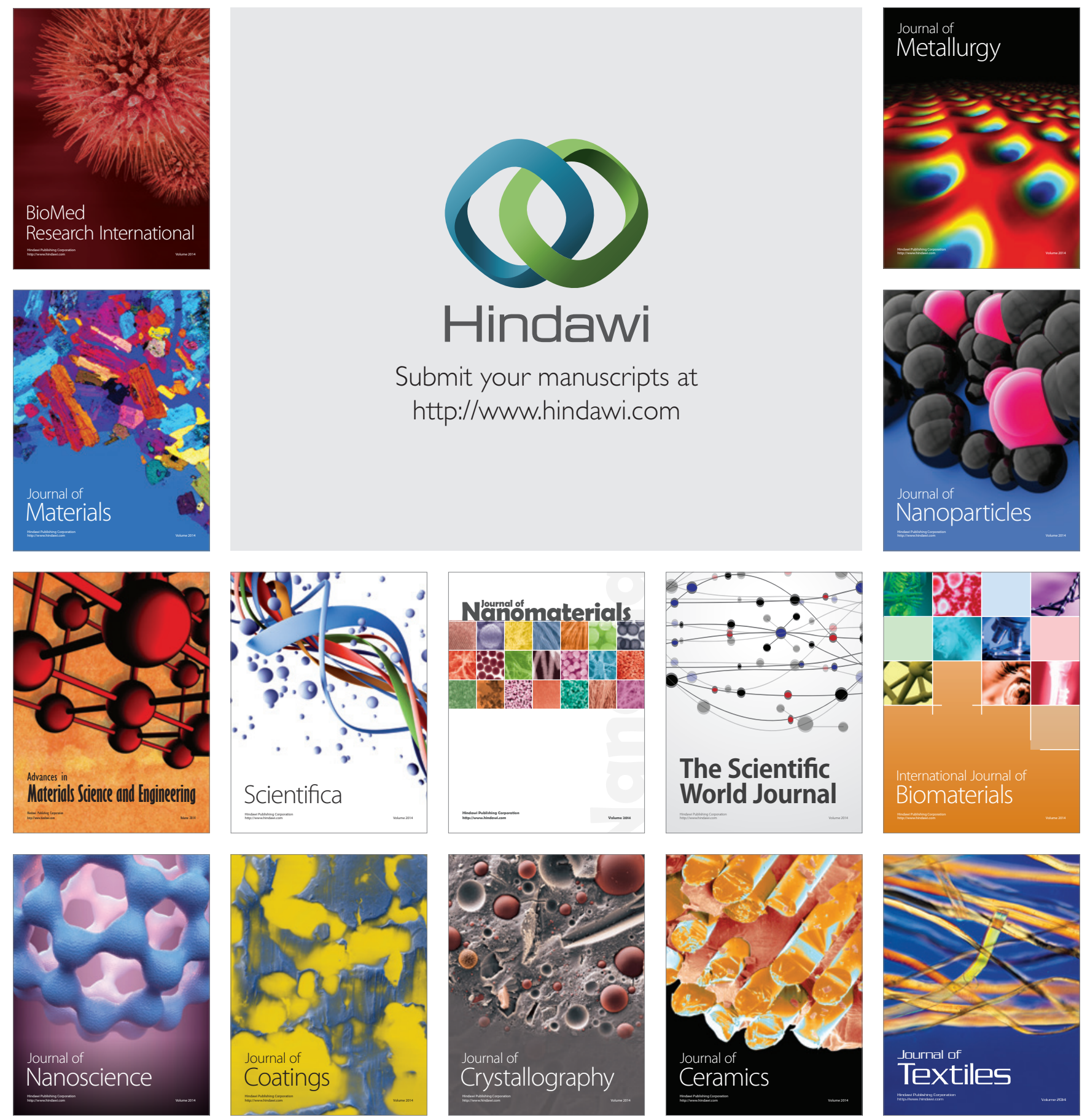